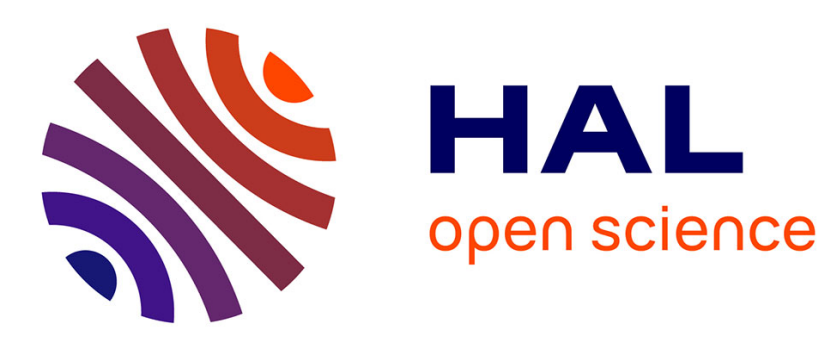

\title{
Competing Activities in Social Networks
}

\author{
Mohamed Belhaj, Frédéric Deroïan
}

\section{To cite this version:}

Mohamed Belhaj, Frédéric Deroïan. Competing Activities in Social Networks. 2010. halshs-00481460

\section{HAL Id: halshs-00481460 \\ https://shs.hal.science/halshs-00481460}

Preprint submitted on 6 May 2010

HAL is a multi-disciplinary open access archive for the deposit and dissemination of scientific research documents, whether they are published or not. The documents may come from teaching and research institutions in France or abroad, or from public or private research centers.
L'archive ouverte pluridisciplinaire HAL, est destinée au dépôt et à la diffusion de documents scientifiques de niveau recherche, publiés ou non, émanant des établissements d'enseignement et de recherche français ou étrangers, des laboratoires publics ou privés. 


\title{
GREQAM
}

Document de Travail

Groupement de Recherche en Economie Quantitative d'Aix-Marseille - UMR-CNRS 6579 Ecole des Hautes Etudes en Sciences Sociales Universités d'Aix-Marseille II et III

\section{Competing Activities in Social Networks}

\author{
Mohamed BELHAJ \\ Frédéric DEROÏAN
}

April 2010 


\title{
Competing activities in social networks
}

\author{
Mohamed Belhaj and Frédéric Deroïan*
}

April 15, 2010

\begin{abstract}
A set of agents is organized in a social network, which conveys synergies in two activities. Each agent has one unit of a resource to allocate between two activities. We show that individual choices are shaped by Bonacich centrality measures and an attractiveness multiplier. The latter, combined with the elasticity of Bonacich centrality with respect to the intensity of interaction, drives the sign of the network reaction to a modification of the costs of activities.
\end{abstract}

JEL: D85, C72.

Keywords: Social Network, Limited Resource, Competing Activities, Attractiveness Multiplier, Elasticity of Bonacich Centrality.

*Mohamed Belhaj: GREQAM and Ecole Centrale de Marseille, mbelhaj@ec-marseille.fr. Frédéric Deroïan: GREQAM, frederic.deroian@univmed.fr. 


\section{Introduction}

In many circumstances, individual behaviors depend on the choice of others in a reference group. This view is supported by a recent empirical literature that emphasizes the role of social networks. Well-known examples of peer effects between neighbors can be found in the workplace, education, job search, criminality, or research activity of academics. ${ }^{1}$ Considering that individuals are organized in a social network, Ballester et al. (2006) have shown that the magnitude of the peer influence exerting on an individual may not be homogenous across the network. In their model, agents exert effort in a costly activity that conveys synergies among neighbors. Under linear-quadratic utilities, an equilibrium exists for sufficiently low level of interaction, and efforts are related to a Bonacich centrality measure. The Bonacich measure captures the network structure, and indicates how 'central' agents are in regard to their position on the network. Indeed, more central agents on the network exert more effort. This result applies when agents undertake one activity. However, individuals are often involved in multiple activities that use the same resource. For instance, students have to share time between education and leisure, and professors between research activity and teaching; farmers have to allocate lands between different plantations, etc. When the available resource is scarce, activities are competing in its use. In this context, the network may favor either of the activities in competition. This issue has not been addressed in the literature.

To proceed, we consider a society in which each agent has one unit of a resource to allocate between two activities 1 and 2. Agents are organized in a social network, through which synergies transit between neighbors along the same activities.

First, this paper studies the impact of the structure of the social network on individual decisions in terms of resource allocation. We show that equilibrium efforts in activity 1 are the sum of effort in autarky and the product of a Bonacich centrality measure by a parameter, that we call Attractiveness Multiplier. The Bonacich centrality is associated with a decay factor, which is in our context the ratio of the sum of synergies over the

\footnotetext{
${ }^{1}$ On networked peer effects in the workplace, see Mas and Moretti (2009), on education see CalvóArmengol et al. (2009), on job search see Topa (2000), on criminality see Gleaser et al. (1996), on research activity of academics see Goyal et al. (2008).
} 
sum of costs of effort. The attractiveness multiplier is related to the attractiveness of activity 1 with respect to activity 2, taking into account both intrinsic preferences and synergies. Importantly, this parameter is independent of the network structure. The impact of the network centrality on individual effort in activity 1 crucially depends on the sign of the attractiveness multiplier. When it is positive, more centrality on the social network enhances the activity 1, otherwise network centrality fosters activity 2 .

Second, we study the network response to a variation of the costs of activities. This issue has been under-explored by the literature. Indeed, in the one-activity setting, the response of the network is unambiguous. In opposite, in the two-activity context, the network may intuitively favor either of the activities, making the response of the network ambiguous. We then consider the effect of a variation of preferences or activity costs on individual efforts, assuming without loss of generality that the exogenous shock is favorable to activity 1 .

When the shock affects preferences, the network responds positively to the shock, and individual efforts in activity 1 increase basically more than in the absence of interaction. We then consider a decrease of the cost of activity 1, or an increase of the cost of activity 2. Such a variation affects the intensity of interaction, and the network may react negatively: following the shock, the variation of efforts are lower than in the absence of interaction. The reason is that decreasing the cost of activity 1 admits two opposite effects on efforts in activity 1: a direct effect, enhancing effort in activity 1, and an indirect effect. Indeed, this generates an increase of the intensity of interaction, which enhances centralities; since the network favors activity 2, this latter effect is favorable to effort in activity 2. We examine how the network affects incentives. For clarity we focus on the case in which activity 2 is relatively more attractive than activity 1.

We show that the network always reacts positively to an increase of the cost of activity 2. However, it reacts negatively to a decrease of the cost of activity 1 for agents whose elasticity of Bonacich centrality with respect to intensity of interaction is relatively high. This elasticity is increasing with the intensity of interaction, but it is non monotonic with regard to link addition, or with regard to centrality (we illustrate the points by the study of all 4-player connected architectures). Nevertheless, subsidizing activity 1 or taxing activity 2 is shown to induce always an increase of all efforts in activity 1 , even if the network reacts negatively. 
The article is organized as follows. Next section presents the model. Section 3 studies equilibria, while section 4 is devoted to the static comparative analysis. Section 5 concludes. The last three sections are appendices. The first one collects proofs, the second presents some basic properties of elasticities of Bonacich centralities, the last one studies Bonacich centralities and their elasticities on all 4-player connected networks.

\section{The model}

Let $\mathcal{N}=\{1,2, \cdots, n\}$ be a set of individuals. Each agent has one unit of a resource (time, space, money) to allocate between two costly activities, say activities 1 and 2 .

The social network. Agents are organized in a social network. Formally, we introduce the set $G$ of all $n \times n$ binary matrices that are symmetric and with null diagonal elements. A social network is then represented by a matrix $g=\left[g_{i j}\right] \in G$. If agents $i$ and $j$ are social neighbors, we set $g_{i j}=1$, otherwise $g_{i j}=0$. We say that $g \leq g^{\prime}$ if and only if, for all $i, j, g_{i j} \leq g_{i j}^{\prime}$. We let $d_{i}(g)=\sum_{j=1}^{n} g_{i j}$ denote the number of agent $i$ 's neighbors, or agent $i$ 's degree, in network $g$. We let $\mu(g)$ denote the inverse of the greatest norm of eigenvalues of $g$.

Utilities. Each agent is endowed with one unit to allocate between two activities. The share of the resource that agent $i$ devotes to activity 1 (resp. activity 2 ) is a real number $x_{i}$ (resp. $y_{i}$ ) in $[0,1]$. We consider that agents' efforts in each activity are proportional to the share of the resource devoted in the related activity. Following Ballester et al. (2006), utilities are linear-quadratic. We impose separability in activities:

$$
u_{i}(x, y ; g)=a_{1} x_{i}-\frac{c_{1}}{2} x_{i}^{2}+a_{2} y_{i}-\frac{c_{2}}{2} y_{i}^{2}+\lambda_{1} \sum_{j} g_{i j} x_{i} x_{j}+\lambda_{2} \sum_{j} g_{i j} y_{i} y_{j}
$$

with $a_{1}, c_{1}, a_{2}, c_{2}>0$, and $\lambda_{1}, \lambda_{2} \geq 0$. Throughout the paper, we will interpret $x_{i}$ as agent $i$ 's effort in activity $1{ }^{2}$ The quantity $\lambda_{q} x_{i} x_{j}, q=1,2$, measures the synergy between two neighbors $i$ and $j$ with regard to activity $q$.

\footnotetext{
${ }^{2}$ This formulation encompasses perceived returns of effort. For instance, in the context of education, Jensen (2010) shows that the returns perceived by individuals affect schooling decisions, and these perceptions may be inaccurate. Interestingly, he finds some support for the hypothesis that students underestimate the returns to education in part because they rely heavily on information on the returns within their own community.
} 
Given that activities are costly, the resource may not be fully exploited if activity costs are high enough. We assume that an isolated agent exploits the full resource, that is, $\frac{a_{1}}{c_{1}}+\frac{a_{2}}{c_{2}} \geq 1$. Hence, $x_{i}+y_{i}=1$. Since externalities are positive, this also implies that socialized agents fully use their resource. The equilibrium effort under autarky is easily shown to be $x_{0}=\frac{a_{1}-a_{2}+c_{2}}{c_{1}+c_{2}}$, and we assume that $\left.x_{0} \in\right] 0,1[$.

We define $v_{i}(x ; g)=u_{i}(x, y ; g)$. Writing $y_{i}=1-x_{i}$ for all $i$, the agent $i$ 's utility becomes:

$$
\begin{gathered}
v_{i}(x ; g)=\left(a_{2}-\frac{c_{2}}{2}+\lambda_{2} d_{i}(g)\right)+\left(a_{1}-a_{2}+c_{2}-\lambda_{2} d_{i}(g)\right) x_{i}-\frac{c_{1}+c_{2}}{2} x_{i}^{2} \\
-\lambda_{2} \sum_{j} g_{i j} x_{j}+\left(\lambda_{1}+\lambda_{2}\right) \sum_{j} g_{i j} x_{i} x_{j}
\end{gathered}
$$

When some agent increases effort in activity 1 , she decreases effort in activity 2 . Hence, externalities are endogenous to agents' effort levels. Basically, $\frac{\partial v_{i}\left(x_{i}\right)}{\partial x_{j}}=g_{i j}\left(\left(\lambda_{1}+\lambda_{2}\right) x_{i}-\right.$ $\left.\lambda_{2}\right)$. That is, increasing agent $j$ 's effort in activity 1 induces a net positive externality to agent $i$ only if the effort of agent $i$ in activity 1 is larger than the threshold $x_{e}=\frac{\lambda_{2}}{\lambda_{1}+\lambda_{2}}$. Second, $\frac{\partial^{2} v_{i}\left(x_{i}\right)}{\partial x_{i} \partial x_{j}}=g_{i j}\left(\lambda_{1}+\lambda_{2}\right) \geq 0$. When some neighbor increases effort in activity 1 , this reinforces incentives to invest in activity $1\left(\lambda_{1}\right)$. Moreover, due to resource constraint, the increase of the neighbor's effort in activity 1 mechanically decreases her effort in activity 2 , which reduces incentives to invest in activity $2\left(\lambda_{2}\right)$. In other words, synergies cumulate.

We let $\gamma=\frac{\lambda_{1}+\lambda_{2}}{c_{1}+c_{2}}$ be the ratio of synergies over costs of effort, we call it the intensity of interaction or equivalently decay factor. Throughout the paper, we assume that $\gamma<$ $\mu(g)$. This relatively low level of interaction guarantees the existence of equilibrium. We define the quantity $m=x_{0}-x_{e}$, that we call Attractiveness Multiplier, which will play a crucial role in our analysis. It is positively related to the attractiveness of activity 1. This parameter takes into account both intrinsic preferences and intensity of synergies between neighbors. It is positive if strategic complementarities in activity 1 are sufficiently high with regard to strategic complementarity in activity 2 , or if the cost of effort in activity 2 is high enough in regard to activity 1 , or if the relative unit intrinsic return of activity 1 vis-à-vis activity 2 is high enough. Actually, we will see thereafter that the sign of $m$ is the sign of the externalities. 


\section{Which activity is promoted by the network?}

Given that the social network conveys synergies, individual decisions will depend on agents' positions on the network. Moreover, as activities are competing, the position on the network will favor only one activity. We examine thereafter how the network shapes efforts, and in particular, whether the network promotes the same activity for all agents or not.

The first order condition of the utility function of agent $i$ writes as follows:

$$
x_{i}^{*}-\gamma \sum_{j} g_{i j} x_{j}^{*}=x_{0}-\frac{\lambda_{2}}{c_{1}+c_{2}} d_{i}(g)
$$

We restrict attention to interior equilibria. ${ }^{3}$ When $\gamma<\mu(g)$, the vector of Bonacich centralities is well-defined and is written $B(g ; \gamma)=\sum_{k=0}^{+\infty} \gamma^{k} g^{k} J$, where $J$ is the vector of ones. Therefore, the individual Bonacich centrality can be expressed as $B_{i}(g ; \gamma)=$ $\sum_{k=0}^{+\infty} \gamma^{k} m_{i}(k ; g)$, with $m_{i}(k ; g)$ being the number of paths (including loops) of length $k$ from agent $i$ to others including herself. It measures the sum of paths of all lengths to others $k$, where the number of paths of length $k$ is weighted by the decay factor $\gamma^{k}$ (see Ballester et al. [2006]). Note that $B_{i}=1$ in the empty network. The next proposition expresses the individual effort as a function of the Bonacich centrality of the agent in the network with decay parameter $\gamma$ :

Proposition 1 The effort in activity 1 of each agent is given by

$$
x_{i}^{*}=x_{0}+m \cdot\left(B_{i}(g ; \gamma)-1\right)
$$

The effort can therefore be expressed as the sum of the effort in autarky, $x_{0}$, plus a (net) network effect. This latter is the product of the attractiveness multiplier by the difference between individual Bonacich measure and 1 (which is the Bonacich measure in the empty network). Thus, the influence of the network can be decomposed in two factors: first, the attractiveness multiplier, that can be either positive or negative, indicates which activity is promoted; second, positions of agents are taken into account

\footnotetext{
${ }^{3}$ This requires that the total synergies on the network are low enough, a sufficient condition is presented in remark 2 below.
} 
through Bonacich centrality measure. ${ }^{4}$ More centrality enhances effort in activity 1 when the attractiveness multiplier is positive, otherwise more centrality enhances activity 2. Furthermore, since the attractiveness multiplier is the same for all agents, the network fosters effort in the same activity for all. Note also that the sign of the attractiveness multiplier is the sign of net externalities.

Remark 1. An immediate implication, related to the monotonicity of Bonacich centrality with respect to link addition (see Ballester et al. [2006]), is that, given any network $g$, adding any link increases either activity 1 or activity 2 for every agent.

Remark 2. In general, high synergies can result in corner solutions. To guarantee $\left.x_{i} \in\right] 0,1[$, individual Bonacich centralities have to be sufficiently low. If $m \geq 0$, we just have to check that $x_{i}^{*}<1$, and using equation (4), we get $B_{i}(g ; \gamma)<\frac{1}{m} \frac{\lambda_{1}}{\lambda_{1}+\lambda_{2}}$. If $m<0$, we check that $x_{i}^{*}>0$ obtains if $B_{i}(g ; \gamma)<\frac{1}{|m|} \frac{\lambda_{2}}{\lambda_{1}+\lambda_{2}}$. Since the Bonacich centralities increase with link addition, and noticing that the Bonacich centrality of the complete network is equal to $\frac{1}{1-\gamma(n-1)}$, an interior solution obtains on any network if $\gamma \leq \frac{1}{n-1}\left[1-|m| \cdot \frac{\lambda_{1}+\lambda_{2}}{\min \left(\lambda_{1}, \lambda_{2}\right)}\right]$.

Remark 3. Note that, while the RHS of the FOC given in equation (3) contains an idiosyncratic component, efforts are expressed as a function of a non weighted Bonacich centrality. This formulation obtains since the idiosyncratic element is proportional to agent $i$ 's degree.

\section{How does social network react to subvention or taxation of activities?}

A policy maker interested in promoting activity 1 can alter $a_{1}$ or $a_{2} \cdot{ }^{5}$ An alternative policy action consists in modifying $c_{1}$ or $c_{2}$, by either subsidizing activity 1 for all agents,

\footnotetext{
${ }^{4}$ This latter factor is presnt in Ballester et al. (2006), the former to our multi-activity setting.

${ }^{5}$ For instance, in the case of education, $a_{1}$ may represent an individual preference for activity 1 ; a state may increase the expected revenue associated with education by favoring the insertion of educated job seekers, or wages, etc. In the example of farmers, $a_{1}$ may correspond to the price of good 1; thus, a state controlling prices can increase the price of good 1 .
} 
or by taxing activity $2 .{ }^{6}$ Importantly, altering costs affects the level of interaction, and therefore, the network structure affects the return of such a policy. How does the social network react to the policy of subvention or taxation?

We will first examine the issue and we will stress the role played by the elasticity of Bonacich centrality with respect to decay. Second, we will explore some properties of the elasticities.

\subsection{How does the network react to policy intervention?}

We suppose without loss of generality a modification of parameters favorable to activity 1 , we consider a marginal increase of parameter $p$, with $p=a_{1},-a_{2},-c_{1}, c_{2}$. This variation results in an increase of $x_{0}$ and $m\left(\frac{\partial x_{0}}{\partial p}>0, \frac{\partial m}{\partial p}>0\right)$. The differentiation of equilibrium effort with respect to any parameter $p$ is decomposed as follows:

$$
\frac{\partial x_{i}^{*}}{\partial p}=\underbrace{\frac{\partial x_{0}}{\partial p}}_{\text {Idiosyncratic Effect }}+\underbrace{\left(B_{i}(g ; \gamma)-1\right) \cdot \frac{\partial m}{\partial p}}_{\text {Network Effect }}+\underbrace{m \cdot \frac{\partial B_{i}(g ; \gamma)}{\partial \gamma} \frac{\partial \gamma}{\partial p}}_{\text {Anteraction Effect }}
$$

The induced variation of effort is the sum of an idiosyncratic effect and a network effect. The former corresponds to the variation of effort in autarky. The latter is shaped by two components: the variation of the attractiveness multiplier and the variation of the centrality. Varying parameters $a_{1}$ or $a_{2}$ entails a positive idiosyncratic effect and a positive network effect (attractiveness effect is positive, and there is no interaction effect), thus the network accentuates the increase of individual efforts in activity 1 with regard to autarky.

We consider now cost variation. For clarity of exposition, we assume $m<0$ (the case $m>0$ is symmetric, see Table 1 for a summary). Consider first an increase of $c_{2}$. The three effects, autarky, attractiveness and interaction effects, are positive. We conclude that efforts in activity 1 increase, and that the increase is larger than that obtained under autarky. Second, consider a decrease of activity 1 . Since $m<0$, the

\footnotetext{
${ }^{6}$ For instance, in case of education versus leisure, a state can lower the cost of effort in education by building bibliothecas or playgrounds, reducing transportation cost to schools, etc. In the case of farmers, a state may subsidize production cost to encourage the adoption of a technology.
} 
interaction effect is negative, and the overall network effect is ambiguous: on one hand activity 1 becomes more attractive, on the other hand the intensity of interaction $\gamma$ is enhanced, increasing Bonacich centralities and then fostering effort in activity 2. Let $\epsilon_{i}(g ; \gamma)=\frac{\gamma}{B_{i}(g ; \gamma)-1} \frac{\partial B_{i}(g ; \gamma)}{\partial \gamma}$ be the elasticity of agent $i$ 's Bonacich centrality (net of centrality in empty network) with respect to the intensity of interaction. This elasticity has a finite value as soon as the Bonacich measure exists, and it tends to infinity as the intensity of interaction approaches its upper limit (see Appendix 1). We obtain:

Proposition 2 Consider $m<0$. Following a decrease of $c_{1}$, the network effect is negative for agents such that $\epsilon_{i}(g, \gamma)>\frac{-x_{0}}{m}$. In contrast, increasing $c_{2}$ induces a positive network effect for all agents, irrespective of the value of the elasticity.

Proposition 2 shows that, when activity 2 is relatively more attractive than activity 1 , i.e. $m<0$, increasing $c_{2}$, the network provides a support to the policy aim, i.e. all agents increase more effort in activity 1 than if they were in autarky. In opposite, decreasing $c_{1}$, the network plays against the policy aim for agents such that the elasticity of Bonacich centrality with respect to decay is sufficiently high. ${ }^{7}$ The following example illustrates the possibility of obtaining negative network reaction for high elasticities.

Regular networks. Let $g_{k}$ denote a regular network of degree $k \in\{0,1, \cdots, n-1\}$, i.e. a network in which every agent has $k$ neighbors. We find $B_{i}\left(g_{k} ; \gamma\right)=\frac{1}{1-k \gamma}$ for all $i$, under the condition that $k<\frac{1}{\gamma}$ (here, $\mu(g)=\frac{1}{\gamma}$ ). We deduce that $\epsilon_{i}\left(g_{k} ; \gamma\right)=\frac{1}{1-k \gamma}$. We define $k_{1}=\frac{a_{1}-a_{2}+c_{2}}{\lambda_{2}}$; note that $m<0$ implies that $k_{1}<\frac{1}{\gamma}$. We obtain that, for any regular network $g_{k}, \mathcal{L}\left(g_{k} ; \gamma\right)=\emptyset$ if $k \leq k_{1}$, while $\mathcal{L}\left(g_{k} ; \gamma\right)=\mathcal{N}$ if $k>k_{1}$. Hence, when decreasing $c_{1}$, the network plays against the policy aim if the network is sufficiently dense. ${ }^{8}$

Despite the network can react negatively, the global effect of the marginal decrease of $c_{1}$ on individual effort in activity 1 , taking into account of both idiosyncratic and network effect, is unambiguous. Indeed, it can be shown that a marginal decrease of the cost of activity 1 (resp. a marginal increase of the cost of activity

\footnotetext{
${ }^{7}$ When $m>0$, subsidizing activity 1 induces a positive network reaction, while taxing activity 2 induces a negative network effect for agents with elasticity higher than $\frac{1-x_{0}}{m}$.

${ }^{8}$ Similarly, when $m>0$, let $\mathcal{L}^{\prime}(g ; \gamma)=\left\{i \in \mathcal{N} / \epsilon_{i}(g ; \gamma)>\frac{1-x_{0}}{m}\right\}$, define $k_{c}^{\prime}=\frac{c_{1}-a_{1}+a_{2}}{\lambda_{1}}$, and note that $m>0$ implies that $k_{c}^{\prime}<\frac{1}{\gamma}$. Then, $\mathcal{L}^{\prime}\left(g_{k} ; \gamma\right)=\emptyset$ if $k \leq k_{c}^{\prime}$, while $\mathcal{L}^{\prime}\left(g_{k} ; \gamma\right)=\mathcal{N}$ if $k>k_{c}^{\prime}$.
} 
2) always enhances individual effort in activity 1 (proof in Appendix 1). In particular, when the interaction effect is negative, the idiosyncratic effect always dominates the network effect.

Thus, a policy maker that wants to promote activity 1 by affecting activity costs should be aware of the possible negative reaction of the social network. This happens when the network favors the activity that is not supported by the policy maker. In that case, taxing the activity that is favored by the network induces a positive reaction of the network for all agents. ${ }^{9}$

INSERT Table 1 here

\subsection{Elasticities and networks}

The previous analysis suggests that the elasticity of Bonacich centralities with respect to decay is crucial to assess how the network reacts to a policy affecting activity costs. We examine now how this elasticity varies with intensity of interaction and we explore some familiar network structures. We let $\mathcal{L}(g ; \gamma)=\left\{i \in \mathcal{N} / \epsilon_{i}(g ; \gamma)>\frac{x_{0}}{|m|}, m<0\right\}$.

Corollary 1 Consider $\gamma, \gamma^{\prime}$, with $\gamma \leq \gamma^{\prime}$. Then, $\mathcal{L}(g ; \gamma) \subset \mathcal{L}\left(g ; \gamma^{\prime}\right)$.

This result is driven by the increase of the elasticity with $\gamma$ (see Appendix 1). Moreover, it is important to emphasize that the properties of the elasticity differ from those of Bonacich centralities. Precisely, there is no monotonic relationship between elasticities and link addition, nor systematic regularity between elasticities and Bonacich centrality. Thus, there is no monotonic relationship between individual effort and the sign of network reaction. We illustrate these points by examining some specific network structures.

\footnotetext{
${ }^{9}$ Our analysis departs from traditional policy concern in presence of strategic interaction. A wellknown consequence of the presence of synergies is coordination problems, because agents have a tendency to conform to the actions of others (Wilson [1987], Bernheim [1994]), which may stick individual and aggregate behaviors to undesirable outcomes (Cooper and John [1988], Akerlof [1997], Brock and Durlauf [2001]). This opens the scope for policy intervention devoted to catalyze individual actions toward a Pareto-improving outcome.
} 
4-player networks. We examine the elasticities of Bonacich centralities on the six 4player connected architectures. Two messages appear. First, the elasticity is not always increasing in link addition (see Table 3, the two numbers in bold for $\gamma=0.05$ ). Second, on a given network, more central agent have not always lower elasticities (see Table 3, the two numbers in bold for $\gamma=0.45$ ).

INSERT Figure 1 here

INSERT Table 2 here

INSERT Table 3 here

\section{Conclusion}

This article has examined the impact of the structure of a social network on individual choices in the allocation of a scarce resource between two activities. One key feature of the model is that the same social network conveys synergies in both activities. We first characterize the individual effort in both activities as a function of a unique Bonacich centrality measure, and network centrality favors the same activity for all agents. A policy maker aiming at promoting activity 1 by subsidizing the cost of activity 1 or taxing activity 2 should take into account the attractiveness multiplier. Indeed, when the network favors activity 2 , choosing to subsidize activity 1 can generate a negative reaction of the network to the policy intervention, limiting policy effect. Such counterproductive network effect occurs when elasticities of Bonacich centralities with respect to the intensity of interaction are relatively large.

It would be interesting to test the predictions of the model. ${ }^{10}$ Further, the theoretical model can be extended in the following directions. One may introduce heterogeneity in

\footnotetext{
${ }^{10}$ In the context of education, Calvó-Armengol, Patacchini and Zénou (2009) find empirical support
} 
individual preferences. One may also examine the endogenous formation of the social network.

\section{Appendix 1: proofs}

Proof of proposition 1. For sufficiently low value of parameter $\gamma$, this linear system (3) is invertible, and there exists a unique equilibrium. Define column-vector $z$ with typical element $z_{i}=x_{i}+m-x_{0}$. Plugging the new variable into equation (3), one obtains $z_{i}-\gamma \sum_{j} g_{i j} z_{j}=m$. Since $\gamma<\mu(g)$, the system $(I-\gamma g) z=m J$ is invertible (see Debreu and Herstein [1953]) and the solution can be written as a Bonacich centrality measure $B(g ; \gamma)=\sum_{k=0}^{\infty} \gamma^{k} g^{k} J$. The individual effort in activity 1 is thus written $x_{i}=x_{0}+m \cdot\left(B_{i}(g ; \gamma)-1\right)$. The existence of interior solutions are guaranteed by hypothesis (see remark 2 for a sufficient condition).

Proof that a marginal decrease of the cost of activity 1 (resp. a marginal increase of the cost of activity 2) always enhances efforts in activity 1 . We differentiate the FOCs given in equation (3) with respect to parameters $c_{1}, c_{2}$. For $d c_{1}<0$, the FOCs are written

$$
\left(c_{1}+c_{2}\right) x_{i}^{*}-\left(\lambda_{1}+\lambda_{2}\right) \sum_{j} g_{i j} x_{j}^{*}=a_{1}-a_{2}+c_{2}-\lambda_{2} d_{i}(g)
$$

Then, $d x^{*}=\left(-\frac{d c_{1}}{c_{1}+c_{2}}\right) \cdot(I-\gamma G)^{-1} x^{*}$, where $(I-\gamma G)^{-1} x^{*}$ is a well-defined weighted Bonacich measure since $\gamma<\mu(g)$ (see remark 1 pp. 1409 in Ballester et al. [2006]), and this quantity is positive since $x^{*}$ is nonnegative. As well, for $d c_{2}>0$, we find $d x^{*}=\left(\frac{d c_{2}}{c_{1}+c_{2}}\right) \cdot(I-\gamma G)^{-1}\left(1-x^{*}\right)$, where $(I-\gamma G)^{-1}\left(1-x^{*}\right)$ is a well-defined weighted Bonacich measure, and this quantity is positive since $1-x^{*}$ is nonnegative. $\diamond$

Proof of proposition 2. Rearranging equation (5), we find that, in case of a marginal

that Bonacich centrality enhances effort in education. Our model provides conditions under which network centrality may to some extent deter education. In particular, in Calvó-Armengol, Patacchini and Zénou (2009), the proxi for effort is performance at school. Our work suggests that if the proxi for effort was instead the duration of activity, then, taking into account that the residual time may also generate utility, the social network may not always enhance education. 
decrease of the cost of activity 1, the network effect is positive if and only if

$$
m \cdot \epsilon_{i}(g ; \gamma) \geq-x_{0}
$$

In case of a marginal increase of the cost of activity 2, the network effect is positive if and only if

$$
m \cdot \epsilon_{i}(g ; \gamma) \leq 1-x_{0}
$$

Note that when $m<0$ inequality (7) holds. The following proposition follows directly.

\section{Appendix 2: basic properties of $\epsilon_{i}$}

We have $\epsilon_{i}(g ; \gamma)=\frac{\gamma}{B_{i}(g ; \gamma)-1} \frac{\partial B_{i}(g ; \gamma)}{\partial \gamma}$. Let us denote $d_{i, q}=\left[g^{q} J\right]_{i}$, for all $q \geq 1$. We denote $d_{i}=d_{i, 1}$, choosing either of the two notations for convenience. Basically,

$$
\epsilon_{i}(g ; \gamma)=\frac{\sum_{q=1}^{\infty} q \gamma^{q} d_{i, q}}{\sum_{q=1}^{\infty} \gamma^{q} d_{i, q}}
$$

- The elasticity is finite if Bonacich centrality is finite. Notice that the Bonacich centrality is defined for all $\gamma<\mu(g)$. Consider some real number $\beta>1$ and such that $\beta \gamma<\mu(g)$. Then we will show that the series $\sum_{q \geq 1} q \gamma^{q} d_{i, q} \leq \sum_{q \geq 1}(\beta \gamma)^{q} d_{i, q}$. It is sufficient to show that there exists some integer $q_{0}$ such that $q \leq \beta^{q}$ for any $q \geq q_{0}$. This means $\frac{1}{\ln (\beta)} \ln (q)<q$ for any $q \geq q_{0}$. This basically holds for some $q_{0}$. $\diamond$

- The elasticity tends to infinity as $\gamma$ tends to $\mu(g)$. We will see that for any real number $A, \lim _{\gamma \rightarrow \bar{\gamma}} \epsilon_{i}(g ; \gamma)>A$. We have:

$$
\epsilon_{i}(g ; \gamma)=\frac{B_{i}-1}{B_{i}}+\frac{B_{i}-\left(1+\gamma d_{i}\right)}{B_{i}}+\frac{B_{i}-\left(1+\gamma d_{i}+\gamma^{2} d_{i, 2}\right)}{B_{i}}+\cdots
$$

Hence, for any integer $\tilde{A}$, we have $\epsilon_{i}(g ; \gamma) \geq \tilde{\epsilon}_{i}$ with

$$
\tilde{\epsilon}_{i}=\frac{B_{i}-1}{B_{i}}+\frac{B_{i}-\left(1+\gamma d_{i}\right)}{B_{i}}+\frac{B_{i}-\left(1+\gamma d_{i}+\gamma^{2} d_{i, 2}\right)}{B_{i}}+\cdots+\frac{B_{i}-\left(\sum_{k=0}^{\tilde{A}} \gamma^{k} d_{i, k}\right)}{B_{i}}
$$

Now, $\tilde{\epsilon}_{i}$ writes:

$$
\tilde{\epsilon}_{i}=\tilde{A}-\frac{\tilde{A}}{B_{i}}-\frac{(\tilde{A}-1) \gamma d_{i}}{B_{i}}-\frac{(\tilde{A}-2) \gamma^{2} d_{i, 2}}{B_{i}}-\cdots-\frac{\gamma^{\tilde{A}} d_{i, \gamma}}{B_{i}}
$$


and since $\lim _{\gamma \rightarrow \bar{\gamma}} B_{i}(g ; \gamma)=+\infty$, we obtain that $\lim _{\gamma \rightarrow \bar{\gamma}} \tilde{\epsilon}_{i}=\tilde{A}$. Hence, taking $\tilde{A} \geq A$, it stems that $\lim _{\gamma \rightarrow \bar{\gamma}} \tilde{\epsilon}_{i}>A$, and the result follows. $\diamond$

- The elasticity is increasing in $\gamma$. We have $\frac{\partial \epsilon_{i}}{\partial \gamma}>0$ if and only if

$$
\left(B_{i}-1\right)\left(\frac{\partial B_{i}}{\partial \gamma}+\gamma \frac{\partial^{2} B_{i}}{\partial \gamma^{2}}\right)-\gamma\left(\frac{\partial B_{i}}{\partial \gamma}\right)^{2}>0
$$

This means

$$
\left(\sum_{k \geq 1} \gamma^{k} d_{i, k}\right)\left(\sum_{k \geq 1} k^{2} \gamma^{k-1} d_{i, k}\right)-\gamma\left(\sum_{k \geq 1} k \gamma^{k-1} d_{i, k}\right)^{2}>0
$$

We remark that coefficients of elements $\left(d_{i, k}\right)^{2}$ are null for all $k$. Concerning the term $d_{i, k} \cdot d_{i, l}$, the coefficient is written $(k+l)^{2} \gamma^{k+l-1}$, and is thus positive. $\diamond$

\section{Appendix 3: 4-player connected architectures}

This appendix presents Bonacich centralities and their respective elasticities with respect to decay - as defined in equation (8) -, for all 4-player connected architectures.

. The circle. For all $i, B_{i}^{\text {Circle }}=\frac{1}{1-2 \gamma}$, and $\epsilon_{i}^{\text {Circle }}=B_{i}$.

. The complete network. For all $i, B_{i}^{\text {Complete }}=\frac{1}{1-3 \gamma}$, and $\epsilon_{i}^{\text {Complete }}=B_{i}$.

. The line network with links $12,23,34$.

$B_{1}^{\text {Line }}=B_{4}^{\text {Line }}=\frac{1+\gamma-\gamma^{2}}{1-3 \gamma^{2}+\gamma^{4}}, B_{2}^{\text {Line }}=B_{3}^{\text {Line }}=\frac{1+2 \gamma-\gamma^{3}}{1-3 \gamma^{2}+\gamma^{4}}$.

$\epsilon_{1}^{\text {Line }}=\epsilon_{4}^{\text {Line }}=\frac{1+4 \gamma+3 \gamma^{2}-4 \gamma^{3}-3 \gamma^{4}+2 \gamma^{5}}{\left(1-3 \gamma^{2}+\gamma^{4}\right)\left(1+2 \gamma-\gamma^{3}\right)}, \epsilon_{2}^{\text {Line }}=\epsilon_{3}^{\text {Line }}=\frac{2+6 \gamma+3 \gamma^{2}-4 \gamma^{3}-3 \gamma^{4}+\gamma^{6}}{\left(1-3 \gamma^{2}+\gamma^{4}\right)\left(2+3 \gamma-\gamma^{2}-\gamma^{3}\right)}$.

. The star network with links $12,13,14$.

$B_{1}^{\text {Star }}=\frac{1+3 \gamma}{1-3 \gamma^{2}}, B_{k}^{\text {Star }}=\frac{1+\gamma}{1-3 \gamma^{2}}$ for $k=2,3,4$.

$\epsilon_{1}^{\text {Star }}=\frac{1+2 \gamma+3 \gamma^{2}}{\left(1-3 \gamma^{2}\right)(1+\gamma)}, \epsilon_{k}^{\text {Star }}=\frac{1+6 \gamma+3 \gamma^{2}}{\left(1-3 \gamma^{2}\right)(1+3 \gamma)}$ for $k=2,3,4$.

. The T network with links $12,23,24,34$.

$B_{1}^{T}=\frac{1+\gamma-\gamma^{2}-\gamma^{3}}{1-4 \gamma^{2}-2 \gamma^{3}+\gamma^{4}}, B_{2}^{T}=\frac{1+3 \gamma+\gamma^{2}-\gamma^{3}}{1-4 \gamma^{2}-2 \gamma^{3}+\gamma^{4}}, B_{3}^{T}=B_{4}^{T}=\frac{1+2 \gamma+\gamma^{2}}{1-4 \gamma^{2}-2 \gamma^{3}+\gamma^{4}}$.

$\epsilon_{1}^{T}=\frac{1+6 \gamma+7 \gamma^{2}-\gamma^{4}+2 \gamma^{5}+\gamma^{6}}{\left(1+3 \gamma+\gamma^{2}-\gamma^{3}\right)\left(1-4 \gamma^{2}-2 \gamma^{3}+\gamma^{4}\right)}, \epsilon_{2}^{T}=\frac{3+10 \gamma+15 \gamma^{2}+8 \gamma^{3}-3 \gamma^{4}-2 \gamma^{5}+\gamma^{6}}{\left(3+5 \gamma+\gamma^{2}-\gamma^{3}\right)\left(1-4 \gamma^{2}-2 \gamma^{3}+\gamma^{4}\right)}, \epsilon_{3}^{T}=\epsilon_{4}^{T}=\frac{2+10 \gamma+14 \gamma^{2}+4 \gamma^{3}-4 \gamma^{4}-2 \gamma^{5}}{\left(2+5 \gamma+2 \gamma^{2}-\gamma^{3}\right)\left(1-4 \gamma^{2}-2 \gamma^{3}+\gamma^{4}\right)}$.

. The Almost complete network with links 12, 13, 23, 24, 34 . 
$B_{1}^{A}=B_{4}^{A}=\frac{1+2 \gamma+\gamma^{2}}{1-5 \gamma^{2}-4 \gamma^{3}}, B_{2}^{A}=B_{3}^{A}=\frac{1+3 \gamma+2 \gamma^{2}}{1-5 \gamma^{2}-4 \gamma^{3}}$.

$\epsilon_{1}^{A}=\epsilon_{4}^{A}=\frac{2+12 \gamma+22 \gamma^{2}+16 \gamma^{3}+4 \gamma^{4}}{\left(2+6 \gamma+4 \gamma^{2}\right)\left(1-5 \gamma^{2}-4 \gamma^{3}\right)}, \epsilon_{2}^{A}=\epsilon_{3}^{A}=\frac{3+14 \gamma+27 \gamma^{2}+24 \gamma^{3}+8 \gamma^{4}}{\left(3+7 \gamma+4 \gamma^{2}\right)\left(1-5 \gamma^{2}-4 \gamma^{3}\right)}$.

\section{References.}

Akerlof, G., 1997, Social distance and social decisions, Econometrica, vol. 65, 10051027.

Ballester, C., A. Calvó-Armengol and Y. Zénou, 2006, Who's who in networks. Wanted: the key player, Econometrica, vol. 74, 1403-1417.

Belhaj, M. and F. Deroian, 2009, Risk Taking, Risk Sharing and Systematic Risk, mimeo.

Bernheim, D., 1994, A theory of conformity, Journal of Political Economy, vol. 102, 841-877.

Bonacich, P., 1987, Power and centrality: a family of measures, American Journal of Sociology, vol. 92, 11701182.

Brock, W. and S. Durlauf, Discrete choice with social interactions, Review of Economic Studies, vol. 68, 235-260.

Calvó-Armengol, A., E. Patacchini and Y. Zénou, 2009, Peer effects and social networks in education, forthcoming in the Review of Economic Studies.

Cooper, R. and A. John, 1988, Coordinating coordination failures in Keynesian models, Quaterly Journal of Economics, vol. 103, 441-464.

Debeu, G., and I. Herstein, 1953, Nonnegative Square Matrices, Econometrica, vol. 21, 597607.

Ghiglino C. and S. Goyal, 2008, Keeping up with the neighbours: social interaction in a market economy, mimeo.

Goyal, S. M. van der Leij and J. Moraga-Gonzalez, 2006, Economics: an emerging small world, Journal of Political Economy, vol. 114, 403-432.

Glaeser, E., B. Sacerdote and J. Scheinkman, 1996, Crime and social interactions, The Quarterly Journal of Economics, vol. 111, 507-48.

Jensen, R., 2010, The (perceived) returns to education and the demand for schooling, forthcoming in the Quaterly Journal of Economics.

Mas, A. and E. Moretti, 2009, Peers at work, American Economic Review, vol. 99, 11245 .

Topa, G., 2001, Social interactions, local spillovers and unemployment, Review of Eco- 
nomic Studies, vol. 68, 261-95.

Wilson, W., 1987, The Truly disadvantaged (Chicago: University of Chicago Press) 
FIGURES AND TABLES.

\begin{tabular}{c||cc|cc} 
& \multicolumn{1}{|c|}{ decrease $c_{1}$} & \multicolumn{2}{c}{ increase $c_{2}$} \\
\hline & $m>0$ & $m<0$ & $m>0$ & $m<0$ \\
\hline Idiosyncratic Effect & $(+)$ & $(+)$ & $(+)$ & $(+)$ \\
Attractiveness Effect & $(+)$ & $(+)$ & $(+)$ & $(+)$ \\
Interaction Effect & $(+)$ & $(-)$ & $(-)$ & $(+)$ \\
Network Effect & $(+)$ & $(+)$ iff $\epsilon_{i}(g ; \gamma) \leq \frac{-x_{0}}{m}$ & $(+)$ iff $\epsilon_{i}(g ; \gamma) \leq \frac{1-x_{0}}{m}$ & $(+)$ \\
Effort in activity 1 & $\nearrow x_{i}$ & $\nearrow x_{i}$ & $\nearrow x_{i}$ & $\nearrow x_{i}$
\end{tabular}

Table 1: Summary

\begin{tabular}{l||lllllllllll}
$\gamma$ & $B^{\text {Circle }}$ & $B_{1}^{\text {Line }}$ & $B_{2}^{\text {Line }}$ & $B_{1}^{\text {Star }}$ & $B_{2}^{\text {Star }}$ & $B_{1}^{T}$ & $B_{2}^{T}$ & $B_{3}^{T}$ & $B_{1}^{A}$ & $B_{2}^{A}$ & $B^{\text {Complete }}$ \\
\hline 0.01 & 1.02 & 1.01 & 1.02 & 1.03 & 1.01 & 1.01 & 1.03 & 1.02 & 1.02 & 1.03 & 1.03 \\
0.05 & 1.11 & 1.05 & 1.10 & 1.15 & 1.05 & 1.05 & 1.16 & 1.11 & 1.11 & 1.17 & 1.17 \\
0.1 & 1.25 & 1.12 & 1.23 & 1.34 & 1.13 & 1.13 & 1.36 & 1.26 & 1.27 & 1.39 & 1.42 \\
0.15 & 1.42 & 1.20 & 1.38 & 1.55 & 1.23 & 1.24 & 1.62 & 1.46 & 1.51 & 1.71 & 1.81 \\
0.2 & 1.66 & 1.31 & 1.57 & 1.81 & 1.36 & 1.39 & 1.97 & 1.74 & 1.87 & 2.18 & 2.50 \\
0.25 & 2.00 & 1.45 & 1.81 & 2.15 & 1.53 & 1.62 & 2.48 & 2.16 & 2.50 & 3.00 & 4.00 \\
0.3 & 2.50 & 1.63 & 2.13 & 2.60 & 1.78 & 1.99 & 3.30 & 2.84 & 3.82 & 4.70 & 10.00 \\
0.35 & 3.33 & 1.89 & 2.55 & 3.24 & 2.13 & 2.69 & 4.84 & 4.14 & 8.43 & 10.62 & \\
0.4 & 5.00 & 2.27 & 3.18 & 4.23 & 2.69 & 4.56 & 8.91 & 7.60 & & & \\
0.45 & 10.00 & 2.87 & 4.17 & 5.98 & 3.69 & 23.71 & 50.48 & 43.12 & & & \\
0.5 & & 4.00 & 6.00 & 10.00 & 6.00 & & & & & & \\
0.55 & & 6.77 & 10.50 & 28.64 & 16.75 & & & & & &
\end{tabular}

Table 2: Bonacich centralities 


\begin{tabular}{l||lllllllllll}
$\gamma$ & $\epsilon^{\text {Circle }}$ & $\epsilon_{1}^{\text {Line }}$ & $\epsilon_{2}^{\text {Line }}$ & $\epsilon_{1}^{\text {Star }}$ & $\epsilon_{2}^{\text {Star }}$ & $\epsilon_{1}^{T}$ & $\epsilon_{2}^{T}$ & $\epsilon_{3}^{T}$ & $\epsilon_{1}^{A}$ & $\epsilon_{2}^{A}$ & $\epsilon^{\text {Complete }}$ \\
\hline 0.01 & 1.02 & 1.02 & 1.02 & 1.01 & 1.03 & 1.03 & 1.02 & 1.03 & 1.03 & 1.02 & 1.03 \\
0.05 & 1.11 & 1.10 & 1.08 & 1.06 & $\mathbf{1 . 1 4}$ & 1.15 & 1.09 & $\mathbf{1 . 1 3}$ & 1.16 & 1.13 & 1.17 \\
0.1 & 1.25 & 1.22 & 1.18 & 1.15 & 1.29 & 1.33 & 1.23 & 1.30 & 1.37 & 1.32 & 1.42 \\
0.15 & 1.42 & 1.36 & 1.30 & 1.27 & 1.45 & 1.54 & 1.42 & 1.51 & 1.66 & 1.60 & 1.81 \\
0.2 & 1.66 & 1.53 & 1.45 & 1.43 & 1.64 & 1.83 & 1.69 & 1.80 & 2.09 & 2.02 & 2.50 \\
0.25 & 2.00 & 1.74 & 1.65 & 1.66 & 1.89 & 2.26 & 2.10 & 2.23 & 2.83 & 2.75 & 4.00 \\
0.3 & 2.50 & 2.01 & 1.91 & 1.97 & 2.21 & 2.93 & 2.78 & 2.91 & 4.37 & 4.28 & 10.00 \\
0.35 & 3.33 & 2.38 & 2.27 & 2.42 & 2.67 & 4.22 & 4.06 & 4.21 & 9.72 & 9.63 & \\
0.4 & 5.00 & 2.92 & 2.80 & 3.13 & 3.39 & 7.64 & 7.47 & 7.63 & & & \\
0.45 & 10.00 & 3.77 & 3.64 & 4.40 & 4.67 & $\mathbf{4 2 . 6 7}$ & 42.52 & $\mathbf{4 2 . 6 8}$ & & & \\
0.5 & & 5.33 & 5.20 & 7.33 & 7.60 & & & & & & \\
0.55 & & 9.18 & 9.04 & 20.97 & 21.24 & & & & & & \\
0.6 & & 34.37 & 34.23 & & & & & & & &
\end{tabular}

Table 3: Elasticities of Bonacich centralities with respect to $\gamma$
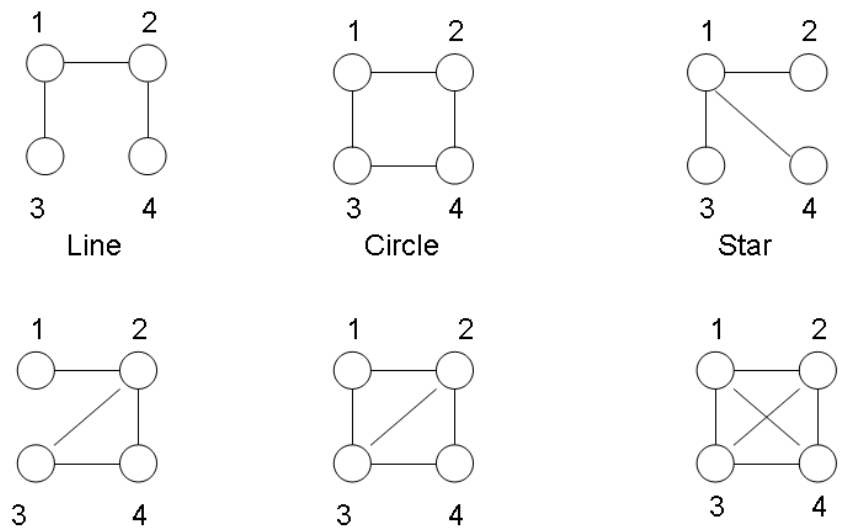

T network

A network

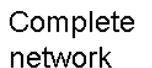

Figure 1: The six 4-player connected architectures 